\title{
EL CARÁCTER PIONERO DE LOS TRABAJOS DE HEINRICH FISCHER $(1875,1881,1882)$ SOBRE LOS JADES SOCIALES EN LA GEOARQUEOLOGÍA DE COSTA RICA
}

\author{
Luis Hurtado de Mendoza ${ }^{1}$, Guillermo E. Alvarado ${ }^{2,3}$ \& Oscar H. Lücke ${ }^{2}$ \\ ${ }^{1}$ Proyecto Hidroeléctrico Toro 3, Instituto Costarricense de Electricidad. \\ ${ }^{2}$ Escuela Centroamericana de Geología, Universidad de Costa Rica. \\ ${ }^{3}$ Área de Amenazas y Auscultación Sísmica y Volcánica (A3SV), \\ Instituto Costarricense de Electricidad.
}

(Recibido: 20/02/07; aceptado: 30/11/07)

\begin{abstract}
Based on a translation into Spanish of a report on jades published in German by H. Fischer in 1882, besides others, an analytical review of his work and results is herein forwarded. The salient pioneering nature for geoarchaeology of Fischer's research is examined within the developing framework of the scientific paradigm in the $19^{\text {th }}$ century. The practical utility of the classification of "social jades" in archaeology, based on criteria such as color and particularly specific gravity, is evaluated. A suggestion is made in favor to follow up studies in the line of Fischer's work, when dealing with lithic artifacts for which it would be inadmissible to apply analytical methods that are destructive.

Keywords: Social jades, mineralogy, geoarchaeology, history of the Geology, Costa Rica.

RESUMEN: Con base en la traducción al idioma español de un informe de H. Fischer publicado en alemán en 1882 y otros trabajos contemporáneos, se ofrece una revisión analítica de su trabajo y resultados concretos. Se destaca la condición pionera de esta investigación en geoarqueología, dentro del contexto paradigmático de la ciencia, que se desarrollaba en el siglo XIX. Se evalúa la utilidad objetiva de la clasificación de los "jades sociales", con base en los criterios de color y, principalmente, gravedad específica. Se sugiere que el método de Fischer amerita seguimiento y perfeccionamiento, de manera que sea aplicado de forma rutinaria en los frecuentes casos de materiales líticos arqueológicos cuya condición particular no admite tratamiento analítico mediante técnicas destructivas.

Palabras clave: Jades sociales, mineralogía, geoarqueología, historia de la Geología, Costa Rica.
\end{abstract}




\section{INTRODUCCIÓN}

El conocido mineralogista de Friburgo, Heinrich Fischer, temprana autoridad en nefrita y jadeíta, publicó en 1875 un compendio de la información sobre estos minerales, incluyendo piezas arqueológicas costarricenses, en su clásico trabajo Nephrit und Jadeit. Las piezas de Costa Rica las obtuvo un coleccionista, el cónsul alemán Johann Friedrich Lahmann, quien amasó centenares de antigüedades costarricenses durante sus muchos años de vivir en el país. Aunque Lahmann permaneció en el país, vendió una parte significativa de su colección a la ciudad de Bremen, para su museo. Es allí en donde Fischer analizó 65 objetos desde 3 puntos de vista: mineralógico, tecnológico y formal artístico.

El informe más completo sobre los jades costarricenses fue publicado por Fischer (1882) en Bremen, Alemania. Comprende 27 páginas y 5 láminas ilustrativas sobre una colección de objetos de jades costarricenses. Un inventario de las piezas analizadas y discutidas en el informe de 1882 , revela un total de 65 objetos, procedentes de diversas partes del país, según se puede ver en el Cuadro 1.

En una reciente clasificación minuciosa de jades de Costa Rica, Aguilar (2003) lo incluye en su lista de referencias con fecha de publicación en 1881 , pero no incorpora los especímenes ilustrados por Fischer en su análisis, de manera que es posible asegurar que el trabajo pionero de Fischer es hasta ahora virtualmente desconocido, e inexplicablemente ha sido pasado por alto en la literatura arqueológica y mineralógica especializada. Lo mismo es cierto en el caso de las otras publicaciones anteriores de Fischer $(1875,1881)$.

En la revisión de la arqueología de Costa Rica y Nicaragua, de Strong (en Steward 1948), el trabajo en jade es brevemente discutido con base en los estudios de Hartman (1901, 1907), Lehmann (1910) y Lothrop (1926). En el caso específico de Costa Rica, Willey (1971) menciona los trabajos de recolección hechos por Minor C. Keith (desde 1886 hasta 1911, probablemente antes) y los informes que suscitó el análisis de aquellos materiales por parte de Lothrop (1926) y Mason (1945).
Cuadro 1

Origen de los jades analizados por Fischer (1882) por regiones y lugares

\begin{tabular}{cccc}
\hline Región & Lugar & $\mathrm{n}$ & $\%$ \\
\hline Cuenca del & Tuis & 1 & 15,4 \\
Reventazón & Agua Caliente & 9 & \\
\hline \multirow{3}{*}{ Valle Central } & Santa Ana & 10 & \\
& Palmares & 9 & 40,0 \\
Guanacaste & San Ramón & 7 & \\
\hline Otros lugares & & 19 & 29,2 \\
\hline Totales & & 10 & 15,4 \\
\hline
\end{tabular}

La revisión histórica de la arqueología de América Central publicada por Stone (1984), incluye una sección dedicada a Costa Rica, en la cual se menciona brevemente los principales logros de Anastasio Alfaro $(1893,1896)$ y Hartman $(1901,1907)$, pero no necesariamente en razón de su involucramiento en estudios del jade. Ninguno de los autores que comparten con Doris Stone el mismo volumen acerca de la Arqueología de América Central, editado por Lange \& Stone (1984), incluye en sus revisiones los trabajos de Fischer, lo que parece confirmar el marcado desconocimiento de los invaluables y pioneros resultados de sus investigaciones de la lapidaria costarricense. Una revisión de la literatura más reciente, no hace más que reafirmar esta impresión. Jones (1998) y Solórzano (2003) se refieren brevemente al trabajo de Fischer. Aguilar (2003) incluye dos referencias en su lista bibliográfica, pero no las discute, y no es citado en trabajos posteriores (p.ej. Salgado \& Guerrero, 2005).

Considerando como pioneros de la arqueología regional centroamericana, a algunos de los viajeros del siglo XIX, cuyos trabajos incluyeron observaciones y registros de materiales provenientes de sitios prehispánicos, con alguna relevancia al caso de Costa Rica, sería menester agregar, en orden cronológico, a los siguientes autores: Stephens (1841), Boyle (1868), Bransford (1884) y Bovallius (1887), pero ninguno de estos se refiere a objetos de jade, en perspectiva científica. 
No es sino hasta el trabajo de Alvarado (2006) en que se trae de nuevo a la luz de la literatura arqueológica y mineralógica, la existencia de dichos trabajos seminales. La razón mayor de todo lo anterior es el hecho de que dichos trabajos fueron publicados en alemán. Sin embargo, una revisión histórica y científica exhaustiva de las conclusiones dadas por Fischer en todos sus alcances, logros, paradigmas de aquel entonces y los actuales, no fue realizada. Es por ello que Lücke y Alvarado se abocaron a traducir del alemán al español el artículo de 1882.

Con base en la versión traducida, el presente trabajo tiene por objetivo realizar un análisis crítico de la publicación del siglo XIX, para que sea en ambos sentidos, incorporada en forma más accesible dentro de la literatura geohistórica y arqueogeológica. Con toda probabilidad, los trabajos en conjunto de Fischer $(1875,1882)$ constituyen el primer estudio de tipo arqueológico y mineralógico detallado y científico de Costa Rica, y por ende, geoarqueológico.

\section{BREVE BIOGRAFÍA DE LEOPOLD HEINRICH FISCHER}

Zoólogo y mineralólogo alemán, nació en Breisgau (Friburgo) el 19 de diciembre de 1817 y murió el 2 de febrero de 1886 en el mismo pueblo. Hijo de d'Aloys Fischer, estudió medicina en Friburgo y también en Viena, Austria. Practicó medicina en Friburgo en Breisgau y a partir de 1845 enseñó mineralogía y zoología. Primero fue asistente y a partir de 1859 fue nombrado profesor. Sus principales obras fueron Orthoptera Europaea (1853), Clavis der Silicate (1864), Chronologischer Überblick über die allmalige Einfuihrung der Mikroskopie in das Studium der Mineralogie, Petrographie und Paläontologie (1868), Kritische mikroscopy Studien (1869, 1871-1873), y Nephrit und Jadeit nach ihren mineralogischen Eigenschaften, sowie nach ihrer urgerschichtlishen und ethnographischen Bedeutung (1875).

Era especialista en clasificación de insectos. Posterior a sus investigaciones en geología, se dedicó al estudio de la antropología y estudió la evolución y distribución geográfica de artefactos de piedra.

\section{EL PARADIGMA ARQUEOLÓGICO EN EL SIGLO XIX}

En cierta forma, los científicos viajeros que llegaron a los países latinoamericanos en el siglo XIX, fueron una consecuencia del espíritu renacentista. Darwin, Stephens, Squier, Hoffman, von Frantzius, Raimondi, Pittier, Lehmann, Sapper y los hermanos Ferraz, entre otros, representaron un afán renovador del quehacer científico. Algunos fueron médicos y otros naturalistas, todos ellos humanistas, imbuidos de una actitud antropocentrista que valoraba el rol del individuo. Opuestos al oscurantismo atávico de la iglesia y de los gobiernos, fueron voceros e impulsores de la educación laica y del desarrollo de la individualidad.

Entendían que no se puede conocer la naturaleza a menos que se la observe directamente y se registren minuciosamente sus características, se comparen y se sistematicen. Estaban alentados por los éxitos logrados por el método experimental y los grandes descubrimientos en física, química, astronomía y medicina. Se guiaban por una actitud crítica y la practicaban.

A pesar de las críticas, el "modelo de las tres edades" (piedra, bronce y hierro) representa el desarrollo de las bases esenciales de la arqueología analítica moderna (Clarke, 1968). Paralelamente, el desarrollo de la geología y la aplicación del concepto de estratigrafía contribuyeron al ordenamiento temporal de los depósitos arqueológicos. En 1847, el arqueólogo danés Worsaae (1821-1885) propuso que el Neolítico en Dinamarca estaba ubicado por el año 3000 a.C., con base en consideraciones estratigráficas, en los resultados de las excavaciones cuidadosas y en los principios de la taxonomía tipológica.

Lo expuesto constituye los antecedentes del trabajo de los arqueólogos pioneros del siglo XIX; el contexto actitudinal, conceptual y metodológico en que hicieron su trabajo. Se debe agregar, sin embargo, que también primó una circunstancia importante, asociada al desarrollo de los museos en Europa y en Norteamérica, y 
que tuvo su reflejo en Costa Rica. Estas instituciones se impusieron la tarea y meta de obtener colecciones de artefactos antiguos de los países del llamado Tercer Mundo, una tendencia que continuó hasta bien entrado el siglo XX. Lo hicieron financiando expediciones de investigación, adquiriendo colecciones de particulares mediante compra, comisionando o alentando a miembros de las misiones diplomáticas, incluso aprovechando la buena disposición de los expatriados que se establecieron en nuestros países.

El mismo Fischer, en una nota de pie de página, nos informa que la colección de objetos de jade que sometió a estudio, es "una pequeña parte de las más de 1000 piezas que contiene la colección de antigüedades costarricenses, las cuales fueron recolectadas en el transcurso de varios años por el Sr. Cónsul Joh. Friedr. Lahmann en San José. La colección entera fue comprada por una asociación de ciudadanos de Bremen por un precio de 10000 marcos alemanes a manera de contribución voluntaria, y fue donada de la manera más generosa a las colecciones estatales de historia natural y etnografía de Bremen en diciembre de 1879”.

\section{METODOLOGÍAAPLICADA POR FISCHER}

Siguiendo una tendencia típica de la época, Fischer considera la muestra de 65 piezas líticas, como obras de arte esculpidas. Reclama la necesidad de someterlas a escrutinio científico, pero en su exposición de propósitos revela su predisposición: "explicar la presencia de algún atributo característico que no se encuentre en ningún otro país, ya sea que involucre el material, la forma o la manera de trabajar las piezas. Al menos debería ser posible a partir de la repetición de formas específicas, encerrar dentro de provincias limitadas a cierto artista o escuelas de arte. Así que esto también es aplicable aquí hasta cierto grado."

Tal actitud, sin embargo, no fue detrimental en absoluto, sino todo lo contrario, pues incorporó al estudio un enfoque interdisciplinario, según veremos a continuación. Fischer, inicia su análisis con las piezas que denomina "hachas planoconvexas". Las somete a examen para intentar dilucidar la técnica de elaboración. Encuentra, en "la parte trasera plana... una superficie lisa aserrada [que muestra] en el medio... una áspera y estrecha banda caracterizada por marcas de fracturas y no por sierra". Deduce de esto que la roca original "fue... trabajada a partir de un bloque mediante una sierra hasta que la unión llegara a ser estrecha y pudiese ser completamente separado fracturándolo".

Las siguientes suposiciones de Fischer, sin duda basadas en las técnicas del escultor, son en extremo interesantes: "El medio para aserrar aparenta ser arena, agua, y un objeto delgado (tendones, filamentos duros de plantas u otro objeto similar, no mediante instrumentos planos. Las superficies son arqueadas, torcidas, si se quiere decir, como si se hubiesen querido a propósito ajustar poco mediante instrumentos planos como hojas de sierra. La fractura final del estrecho puente de unión no es violenta, sino interponiendo cuñas de madera y luego siendo sumergido en agua; bajo esta suposición, es posible aceptar que el artista trabajó primero en la base compacta de toda la pieza de roca y luego aserró la figura; sin embargo, todas las estrechas figuras de roca estarían después de todo en peligro durante el aserrado. Por lo tanto, debió haber sido más probable que la forma general de la escultura y el trabajo con la sierra estuviesen terminados antes de que el artista comenzara con la elaboración del grabado de las líneas."

Fischer opina que la técnica de perforaciones para suspender las piezas mediante la ayuda de un mecate, no difiere de la técnica que ha observado en artefactos equivalentes de México. Reconoce que tales perforaciones se presentan en tres modalidades: horizontales, verticales e inclinadas, a veces aisladas, en otras se combinan dos y hasta tres de estas. Cuando grafica estas perforaciones, ilustra adecuadamente la práctica de ataque desde dos extremos hacia el centro de la pieza.

Otro procedimiento aplicado por Fischer, es que se concentra en la identificación de las materias primas utilizadas. Reconociendo que no son posibles las técnicas conocidas de análisis mineralógico, que implican daño físico a las piezas, intenta acercamientos preliminares que resultan en la proposición de categorías con grados de certeza relativa. Para esta tarea se ayudó con la "Guía Internacional de Colores" de Radden. Reconoce la calcedonia, la jadeíta, la arcilla, la actilonita, el esquisto micáceo y la dolerita; 
pero manifiesta duda en el caso de otros ocho materiales. A nuestro parecer, esta aparente limitación no deja de revelar un aspecto importante y trascendente: la notable diversidad de materias primas líticas (22 categorías) dentro de una colección de origen costarricense.

Por otro lado, está claro que cuando las piezas son pequeñas y pulidas, y no se pueden quebrar, moler o hacerles sección delgada para practicarles otros análisis más específicos y cualitativos, la clasificación petrográfica puede ser limitada, por no decir que subjetiva y hasta errónea (ver comentarios de Alvarado, 2006).

Dentro de estas limitantes, los arqueólogos, aún continúan la práctica de incluir muchos tipos de piedras duras esculpidas en la categoría genérica de "jades" o los llamados "jades sociales o culturales". Según parece, Fischer trató esmeradamente de discernir la diversidad de materiales, que reporta, con la ayuda de la técnica no destructiva que permite determinar la gravedad específica de las rocas. Por ello no deja de ser sorprendente que él, no solo tratara de clasificar cada espécimen petrográficamente (situación que se ha dejado de hacer en los estudios arqueológicos recientes), sino que además, siendo un experto mineralogista respetara, sin embargo, el valor cultural de las piezas, y aplicara las llamadas técnicas no destructivas, hoy día en boga.

Fischer encuentra sorpresiva la presencia de la jadeíta y otros "tenaces minerales con dureza similar al cuarzo", dado que no disponía de información acerca de fuentes naturales en el Nuevo Mundo, de manera que recomienda explorar la posibilidad de su obtención en Asia, vía comercio, a través de los conquistadores españoles. Otro aspecto muy interesante para la época, es que no menciona la presencia de nefrita sino solo de jadeíta. La nefrita se encuentra aflorando en cantidades comerciales solo en Sudamérica o en los Estados Unidos, y en muchos trabajos de la segunda mitad del siglo XX e inicios del XXI se le continúa mencionando a ambos como componentes de los jades, sin percatarse de las implicaciones sobre su procedencia (ver discusión en Alvarado, 2006). Se ha propuesto a Guatemala como la fuente geológica más cercana de la jadeíta (p. ej. Foshang, 1947; Bishop et al., 1985; Fields \& Reents-Budet, 1992; Harlow, 1993; Bishop \& Lange, 1993).
En el lado positivo, es importante el señalamiento de Fischer en cuanto a que sus exámenes de las piezas arqueológicas lo conducen a la convicción de que, en casi todos los casos, los artesanos hicieron su trabajo a partir de cantos rodados, cuya forma era seleccionada en razón del tipo de objeto que se deseaba producir. Esto resulta interesante para la época, dado que nos habla de cantos rodados, que bien pudieron haber sido producidos por acción fluvial o marina. Sin embargo, también es plausible lo contrario, que el artesano se adecuara a la forma y tamaño del canto redondo que en cada momento tuviera disponible. Las piedras duras mencionadas, no parecen ser abundantes ni menos ubicuas. Su rareza era, sin duda, uno de los factores principales que las hacían (y las hacen) apreciables.

Para efectuar clasificaciones, descartó los criterios de procedencia geográfica-arqueológica y de constitución mineralógica de las materia prima. Previó dificultades en razón de la calidad mueble de los artefactos y de las materias primas. Prefirió ceñirse a un "criterio de clasificación más objetivo, el cual es la naturaleza del trabajo artístico que presentan las piezas".

Especificó tres categorías principales: 1) Herramientas y similares (13 objetos), 2) ornamentos o cuentas de collar (21 objetos), y 3) colgantes, perforados para pasar un cordel (22 objetos), así como no clasificados (9 objetos).

Las categorías designadas bajo los términos "ornamentos" y "colgantes" coinciden con las clasificaciones convencionales de jades en arqueología, con la calificación de que tal función, asociada a la definición de rango, fue la más probable durante la vida de los usuarios. Varían los mismos objetos a la función de ofrenda funeraria al ocurrir el deceso de sus poseedores. Por otro lado, la categoría denominada "herramientas" parece implicar funciones utilitarias, a menos que las formas que las sugieren como tales sean meramente emblemáticas. Desafortunadamente, el contexto original de las piezas estudiadas por Fischer, es desconocido.

La observación directa de los especímenes y los datos de gravedad específica obtenidos mediante pruebas de laboratorio, permitieron a Fischer una interesante clasificación de la muestra, por materia prima lítica. 
Al menos 22 tipos de rocas y minerales están representados en la muestra examinada por Fischer (Cuadro 2). Sin perjuicio de la notable diversidad de tipos, resalta la concentración del $57 \%$ de los especímenes en solamente cinco categorías: jadeíta, cuarzo, ópalo, calcedonia y esquisto micáceo.

Alvarado (2006) explica que la jadeíta, el esquisto micáceo y varios tipos de rocas ígneas mencionadas por Fischer, deben de ser de proce- dencia foránea respecto de Costa Rica. Fischer debe haber confundido algunas rocas que analizó y que son originarias de Costa Rica con fonolitas. La fonolita es un tipo de roca volcánica alcalina (minerales tales como sanidina, anortoclasa, nefelina o leucita, entre otros), color verdosa, que suena al golpearlas con el martillo geológico, de allí el nombre. No se conoce hasta el momento en América Central, pero es abundante en lugares de vulcanismo continental o subcontinetal no orogé-

Cuadro 2

Identificación mineralógica de los jades de Costa Rica efectuada por H. Fischer (1882)

\begin{tabular}{|c|c|c|c|c|}
\hline \multirow[t]{2}{*}{ Materia prima } & & & \multicolumn{2}{|c|}{ Gravedad específica } \\
\hline & $\mathrm{n}$ & $\%$ & Promedio & 口 \\
\hline Jadeíta? & 13 & 20,0 & 3,31 & 0,04 \\
\hline Cuarzo & 7 & 10,8 & 2,65 & 0,06 \\
\hline Ópalo & 7 & 10,8 & 2,17 & 0,12 \\
\hline Cuarzo calcedonia & 5 & 7,7 & 2,57 & 0,04 \\
\hline Esquisto micáceo & 5 & 7,7 & 2,78 & 0,12 \\
\hline Pirita & 3 & 4,6 & 4,78 & \\
\hline Gabro serpentina? & 2 & 3,1 & 2,83 & \\
\hline Arcilla & 2 & 3,1 & 1,99 & 0,3 \\
\hline Dolerita & 2 & 3,1 & 2,84 & \\
\hline Fonolita? & 2 & 3,1 & 2,50 & \\
\hline Cuarzo heliotropo & 2 & 3,1 & 2,51 & 0,05 \\
\hline Sanidina? & 2 & 3.1 & 2,55 & \\
\hline Gabro con diálaga verde & 1 & 1,5 & 2,78 & \\
\hline Prenhita-muscovita & 1 & 1,5 & 3,00 & \\
\hline Strahlstein & 1 & 1,5 & 2,95 & \\
\hline Fuchsita & 1 & 1,5 & n.d. & \\
\hline Barnlita & 1 & 1,5 & 2,94 & \\
\hline Serpentina & 1 & 1,5 & 2,86 & \\
\hline Muscovita cromiforme & 1 & 1,5 & 2,75 & \\
\hline Nontronita ?? & 1 & 1,5 & 2,27 & \\
\hline Arcillolita silicificada & 1 & 1,5 & 1,95 & \\
\hline Andesita con anfíboles & 1 & 1,5 & 2,72 & \\
\hline No identificada & 3 & 4,6 & -- & \\
\hline Totales & 65 & 100,0 & & \\
\hline
\end{tabular}


nico, tales como Eifel y Keiserstuhl en Alemania. De allí puede venir la confusión. Probablemente, se trataba de un basalto verdoso. Es probable que Fischer no tenía los elementos suficientes para discernir entre una fonolita de otros tipos de rocas ígneas propias del país.

La figura 1, muestra los resultados de la comparación de rangos de los valores de gravedad específica, por categorías petrográficas y mineralógicas, en los casos en que ha sido posible obtener estos datos estadísticos. Nótese la importante separación entre la jadeíta y los demás tipos, hecho que todavía es utilizado como un criterio para diferenciar esta roca (jadeitita) o su mineral constituyente (jadeíta), usualmente verdosa y muy densa, de otras rocas y minerales de apariencia similar (ver Laguna \& Kussmaul, 1982; Alvarado, 2006).

En conclusión, los trabajos de Fischer fueron pioneros en Costa Rica desde el punto de vista arqueológico y mineralógico, en el sentido de describir con elegancia las piezas precolombinas, destacar su arte, técnicas lapidarias, motivos (humanos, animales, abstractos), acabados, materia prima (tipo de rocas y minerales), número de colección, y por el hecho de aplicar técnicas no destructivas (peso específico, lupa, raya en ocasiones, color, brillo).

\section{REFERENCIAS}

AGUILAR, C.H., 2003: El Jade y el Chamán.155 págs.- Editorial Tecnológica de Costa Rica, Cartago.

ALFARO, A., 1893: Arqueología Costarricense.El Centenario. 4: 241-246.

ALFARO,A., 1896: Antigüedades de Costa Rica.Tipografía Nacional, San José.

ALVARADO, G.E., 2006: Objetos líticos precolombinos en Costa Rica: Tipos, frecuencia petrográfica, materia prima y aspectos

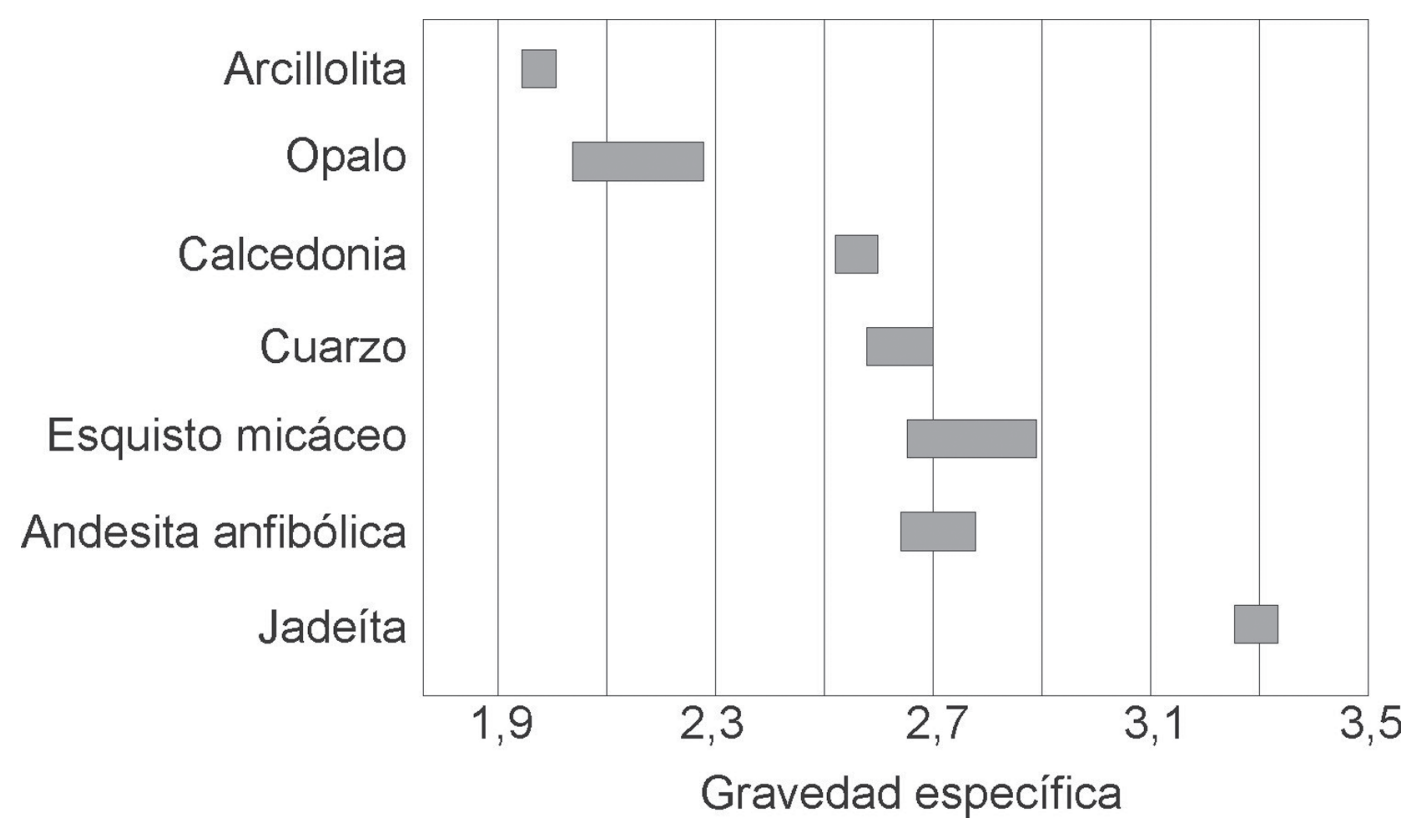

Figura 1: Comparación de la gravedad específica de algunos materiales arqueológicos identificados, según datos aportados por Fischer (1882). 
sobre su utilización.- En: FERNÁNDEZ, P. \& ALVARADO, G.E.: Artesanos y Piedras: Herramientas y escultura precolombina en Costa Rica.- Fundación Museos del Banco Central: 70-127.

BISHOP, R. \& LANGE, F., 1993: Sources of Maya and Central American Jadeites. Data Bases and Interpretations, A Summary.En: LANGE, F. (ed.): Precolumbian Jade, New Geological and Cultural Interpretations: 125-140.

BISHOP, R., SAYRE, E. \& VAN ZEITS, L., 1985: Characterization of Mesoamerican Jade.- En: Application of Science in Examination of Works of Art. Museum of Fine Arts Research Laboratory: 151-156.

BOVALLIUS, C.E.A., 1887: Resa i CentralAmerika 1881-1883.- Almqvist \& Wiksells boktryckeri, Upsala.

BOYLE, F., 1868: A Ride Across a Continent.Londres.

BRANSFORD, J.F., 1884: Report on Explorations in Central America in 1881.- Annual Report of the Board of Regents of the Smithsonian Institution for 1882: 803-825.

CLARKE, D.L., 1968: Analytical Archaeology.Methuen \& Co. Ltd.

FIELDS, V.M. \& REENTS-BUDET, D.J., 1992: Historical Implications of the Jade Trade Between the Maya Lowlands and Costa Rica during the Early Classic Period.- En MARKEL, S. (ed.): The World of Jade.Marg Publications: 81-88.

FISCHER, H., 1875: Nephrit und Jadeit, mineralogisch und ethnographisch u.s.w. [Nefrita y jadeíta, mineralógica y etnográfica etc.]Stuttg. 1875. 8. Schweizerbart, mit 131 Holzschnitten und 2 chromolith. Tafeln; 2. vermehrte Ausgabe 1880; ferner im Archiv f. Anthropologie v. Ecker und Lindernschmit: die Mineralogie als Hilfswissenschaftf. Arch. und Ethnogr. 1879. Bd. XII und XIII; über Amazonenstein, Bd. XII. 1879; über Timur`s Grabmal Bd. XII; in den Mitteilungen d. anthrop. Ges. z. Wien, Bd. VIII. 1878. Mineralog. archaeol. Studien u.s.w.

FISCHER, H., 1881: Bericht über eine Anzahl Steinsculpture aus Costa Rica.Abhandlungen Herausgegeben vom Naturwissenschaftlichen Verein zu Bremen, 7: 152-175.

FISCHER, H., 1882: Bericht über eine Anzahl SteinsculpturenausCostarica.-Abhandlungen ausgegeben vom naturwissenschaftlichen Verein zu Bremen, 7: 153-185.

FOSHANG, W., 1947: Mineralogical Studies of Guatemalan Jade.- Smithsonian Miscelaneous Collection, 145 (5).

HARLOW, G., 1993: Middle American Jade: Geologic and Petrologic Perspectives on Variability and Source.- En: Precolumbian Jade, New Geological and Cultural Interpretations: 9-29.

HARTMAN, C.V., 1901: Archaeological Researches in Costa Rica.- The Royal Ethnographical Museum, Stockholm.

HARTMAN, C.V., 1907: Archaeological Researches on the Pacific Coast of Costa Rica.- Memoirs of the Carnegie Museum, Pittsburgh, 3 (1).

JONES, J., 1998: Jade in Ancient Costa Rica.- The Metropolitan Museum of Art, New York.

LAGUNA, J. \& KUSSMAUL, S., 1982: Análisis de la Colección de Jades del Instituto Nacional de Seguros.- Museo del Jade, INS, San José.

LANGE, F.W. \& STONE, D. (eds.), 1984: The Archaeology of Lower Central America.A School of American Research Book, University of New Mexico Press, Albuquerque. 
LEHMANN, W., 1910: Ergebnisse einer Froschungereise in Mittelamerika und Mexico.-Zeitung fur Ethnologie, 42: 687-749.

LOTHROP, S.K., 1926: Pottery of Costa Rica and Nicaragua.- Museum of the American Indian, Heye Foundation, New York.

MASON, J.A., 1945: Costa Rican Stonework: The Minor Keith Collection.- Anthropological Papers of the American Museum of Natural History, 39, Part 3. New York.

SALGADO, S. \& GUERRERO, J.V., 2005: La distribución de la jadeíta en Centroamérica y su significado social.- Cuadernos de Antropología, 15: 53-64.

SOLÓRZANO, J.C., 2003: Reflexiones en torno a la historiografía y la arqueología en Costa Rica durante el siglo XIX.- En: PERALDO, G. (ed.): Ciencia y Técnica en la Costa Rica del Siglo XIX.- Ed. Tecnológica de Costa Rica, Cartago: 239-264

STEPHENS, J.L., 1841: Incidents of Travel in Central America, Chiapas and Yucatan.Harper and Bros., Nueva York.

STEWARD, J.H., (ed.), 1948: Handbook of South American Indians.- Bureau of American Ethnology, Smithsonian Institution, Washington, D.C, Bulletin 143.

STONE, D., 1984: A History of Lower Central American Archaeology.- En: LANGE, F. \& STONE, D., (eds.): The Archaeology of Lower Central America.- University of New Mexico Press, Albuquerque: 13-32.

WILLEY, G.R., 1971: An Introduction to American Archaeology, Volume 2: South America.- Prentice-Hall, Englewood Cliffs, New Jersey. 